\title{
Three-nucleon force effects in nucleon-deuteron scattering at backward angles
}

\author{
Souichi Ishikawa ${ }^{\star}$ \\ Hosei University, Tokyo, Japan \\ $\star$ ishikawa@hosei.ac.jp \\ Proceedings for the 24th edition of European Few Body Conference, \\ Surrey, UK, 2-6 September 2019 \\ doi:10.21468/SciPostPhysProc.3
}

\begin{abstract}
Elastic and breakup cross sections in nucleon-deuteron scattering at intermediate energies are calculated using a realistic two-nucleon potential and three-nucleon potentials based on the exchange of two pions among three nucleons. Attractive effects of the pion-exchange at medium range region in the three-nucleon potential are recovered by using a shorter range cutoff. It is shown that these effects increase the cross sections at backward angles, which tends to reduce discrepancies between theoretical calculations and experimental data.
\end{abstract}

(c) (1) Copyright S. Ishikawa.

This work is licensed under the Creative Commons

Attribution 4.0 International License.

Published by the SciPost Foundation.
Received 14-10-2019

Accepted 29-11-2019

Published 27-02-2020

doi:10.21468/SciPostPhysProc.3.054

\section{Introduction}

The necessity of three-nucleon potentials (3NPs) in the nuclear Hamiltonian in addition to two-nucleon potentials (2NPs) is well recognized. However our understanding of 3NPs is quite incomplete except the 3NP based on Fig. 1, namely two-pion exchange process among three nucleons $(2 \pi \mathrm{E})$, and then combinations of a $2 \mathrm{NP}$ model and a $2 \pi \mathrm{E}-3 \mathrm{NP}$ model have been often used in few-nucleon calculations.

In spite of various successful $2 \pi \mathrm{E}-3 \mathrm{NP}$ effects on $3 \mathrm{~N}$ observables, there are still unsolved discrepancies between experimental data and calculations. A typical example is the differential cross sections at backward angles of nucleon-deuteron (Nd) elastic scattering for intermediate energy.

It is noticed that the $2 \pi \mathrm{E}-3 \mathrm{NP}$ has quite large attractive character, which should be suppressed by introducing a cutoff procedure to reproduce three-nucleon $(3 \mathrm{~N})$ binding energies. Since it turns out that the range of the cutoff is not limited to a short range, effects of the pionexchange process in 3NP may be eliminated even at rather longer distances. Thus, this ad hoc cutoff procedure might affect $3 \mathrm{~N}$ observables such as ND elastic cross sections at backward angles. In this work, $\mathrm{Nd}$ cross sections are studied using Hamiltonian models consisting of a 
two-nucleon potential and $2 \pi \mathrm{E}-3 \mathrm{NPs}$ with a rather short range cutoff by adding phenomenological repulsive 3 NPs as a counterpart [1].

In Sec. 2, a short note on numerical calculations and nuclear interaction models used in this work will be given. Results of calculations will be presented in Sec. 3, and conclusion will be given in Sec. 4.

\section{Method and models}

\subsection{Three-nucleon Faddeev calculations}

Three-body calculations in the present paper are performed by solving the Faddeev equations [2] as integral equations in coordinate space using a method developed in Ref. [3].

$3 \mathrm{~N}$ partial wave states for which $2 \mathrm{NPs}$ and 3NPs act, are restricted to those with total twonucleon angular momenta $j \leq 6$ for bound state calculations, and $j \leq 5$ for scattering state calculations. For scattering state calculations, $3 \mathrm{~N}$ partial wave states with the total $3 \mathrm{~N}$ angular momentum $J \leq 27 / 2$ are taken into account, while 3NPs are switched on for $3 \mathrm{~N}$ states with $J \leq 13 / 2$.

\subsection{Two-pion exchange three-nucleon potential}

The momentum space representation of a component of the $2 \pi \mathrm{E}-3 \mathrm{NP}$ described by the diagram, Fig. 1, is given by

$$
\begin{aligned}
\hat{V}^{(3: 1,2)}\left(\vec{q}_{1}, \vec{q}_{2}\right)= & \frac{1}{(2 \pi)^{6}}\left(\frac{g_{A}}{2 f_{\pi}}\right)^{2} \frac{1}{\vec{q}_{1}^{2}+m_{\pi}^{2}} \frac{1}{\vec{q}_{2}^{2}+m_{\pi}^{2}}\left(\vec{\sigma}_{1} \cdot \vec{q}_{1}\right)\left(\vec{\sigma}_{2} \cdot \vec{q}_{2}\right) \\
& \times\left[\left(\vec{\tau}_{1} \cdot \vec{\tau}_{2}\right)\left\{a+b\left(\vec{q}_{1} \cdot \vec{q}_{2}\right)\right\}+\left(i \vec{\tau}_{3} \cdot \vec{\tau}_{1} \times \vec{\tau}_{2}\right)\left(i \vec{\sigma}_{3} \cdot \vec{q}_{1} \times \vec{q}_{2}\right) d\right],
\end{aligned}
$$

where momentum $\vec{q}_{i}(i=1,2)$ is defined in Fig. $1, m_{\pi}$ is the pion mass, $\vec{\sigma}_{i}\left(\vec{\tau}_{i}\right)$ is spin (isospin) operator of the nucleon $i, g_{A}$ is the axial charge of the nucleon, and $f_{\pi}$ is the pion decay constant. The coefficients, $a, b$, and $d$, characterize the $2 \pi \mathrm{E}-3 \mathrm{NP}$, which are extracted from pion-nucleon scattering amplitudes. In this paper, I will use a newer version of the Brazil $2 \pi \mathrm{E}-3 \mathrm{NP}, \mathrm{BR}-\mathcal{O}\left(q^{4}\right)$ in Ref. [4] (BR07), whose coefficients are

$$
(a, b, d)=\left(0.981 m_{\pi}^{-1},-2.617 m_{\pi}^{-3},-0.854 m_{\pi}^{-3}\right) .
$$

The coordinate space representation of the $2 \pi \mathrm{E}-3 \mathrm{NP}$ is given by

$$
V^{(3: 1,2)}\left(\vec{r}_{13}, \vec{r}_{23}\right)=\int d \vec{q}_{1} d \vec{q}_{2} e^{-i \vec{q}_{1} \cdot \vec{r}_{13}-i \vec{q}_{2} \cdot \vec{r}_{23}} F_{\Lambda}\left(\vec{q}_{1}^{2}\right) F_{\Lambda}\left(\vec{q}_{2}^{2}\right) \hat{V}^{(3: 1,2)}\left(\vec{q}_{1}, \vec{q}_{2}\right),
$$

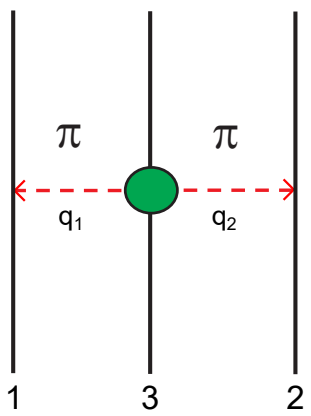

Figure 1: Diagram that shows a component of the $2 \pi \mathrm{E}-3 \mathrm{NP}$. Total $2 \pi \mathrm{E}-3 \mathrm{NP}$ is the sum of cyclic permutations of this diagram. 
Table 1: Empirical and calculated values of the ${ }^{3} \mathrm{He}$ binding energy and strength parameter $V_{\mathrm{C}}$ of the additional C-3NP, Eq. (4), for models used in this paper. See the text for the description of the models.

\begin{tabular}{|c|c|c|}
\hline Model & $\begin{array}{c}V_{\mathrm{C}} \\
(\mathrm{MeV})\end{array}$ & $\begin{array}{c}\mathrm{BE}\left({ }^{3} \mathrm{He}\right) \\
(\mathrm{MeV})\end{array}$ \\
\hline Empirical & & 7.718 \\
\hline AV18 & & 6.928 \\
\hline$+\mathrm{BR}_{660}$ & & 7.763 \\
\hline$+\mathrm{BR}(\mathrm{C})_{1000}$ & 139 & 7.727 \\
\hline$+\mathrm{BR}_{[\mathrm{I}] 0.87}$ & & 7.718 \\
\hline$+\mathrm{BR}(\mathrm{C})_{[\mathrm{I}] 0.5}$ & 159 & 7.726 \\
\hline$+\mathrm{BR}_{[\mathrm{II}] 1.29}$ & & 7.719 \\
\hline$+\mathrm{BR}(\mathrm{C})_{[\mathrm{II}] 0.75}$ & 160 & 7.716 \\
\hline
\end{tabular}

where $\vec{r}_{i j}=\vec{r}_{i}-\vec{r}_{j}$, and $F_{\Lambda}\left(\vec{q}^{2}\right)$ is a form factor. I this work, I will take a dipole form:

$$
F_{\Lambda}\left(\vec{q}^{2}\right)=\left(\frac{\Lambda^{2}-m_{\pi}^{2}}{\Lambda^{2}+\vec{q}^{2}}\right)^{2} .
$$

Here, the parameter $\Lambda$ represents a scale of the cutoff momentum of the exchanged pions. Calculations with the BR07-3NP adapting the dipole form factor with the cutoff parameter $\Lambda$ will be denoted as $\mathrm{BR}_{\Lambda}$.

In the present paper, I will use the Argonne $\mathrm{V}_{18}$ (AV18) 2NP model [5]. Calculated ${ }^{3} \mathrm{He}$ binding energy for the AV18 are shown in Table 1, which indicates the AV18 calculation underbinds the ${ }^{3} \mathrm{He}$ bound state by about $0.8 \mathrm{MeV}$. When the BR07-3NP is combined with the AV18-2NP, the cutoff parameter $\Lambda$ determined to reproduce the ${ }^{3} \mathrm{He}$ binding energy is $\Lambda=660$ $\mathrm{MeV}\left(\mathrm{BR}_{660}\right.$ ) (see Table 1 for numerical values). This value of $\Lambda$ is quite small compared with ones used in one-boson exchange 2NP models, such as the CD-Bonn potential [6], which are larger than $1000 \mathrm{MeV}$. This indicates that some medium range contributions in $2 \pi \mathrm{E}-3 \mathrm{NP}$ may be reduced.

\section{Calculations}

\subsection{Elastic cross sections}

In Fig. 2 (a) and (b), calculations of differential cross section for elastic proton-deuteron ( $p$ - $d$ ) scattering at $E_{p}=65 \mathrm{MeV}$ and $E_{p}=170 \mathrm{MeV}$, respectively, are compared with experimental data $[7,8]$. In the figures, $\mathrm{AV} 18$ and $\mathrm{AV} 18+\mathrm{BR}_{660}$ calculations are shown by black and red curves, respectively, which demonstrates that the deviations between the AV18 calculations and the data, especially at the minimum region, $90^{\circ}<\theta<150^{\circ}$, are well reduced by introducing the $\mathrm{BR}_{660}$ for $E_{p}=65 \mathrm{MeV}$, but is not enough for $E_{p}=170 \mathrm{MeV}$.

Here, I remark that the momentum transfer from the incident proton to deuteron, which is calculated from the scattering angle, exceeds the value of $660 \mathrm{MeV} / \mathrm{c}$ for scattering angles larger than $120^{\circ}$ for $E_{p}=170 \mathrm{MeV}$. Thus, effects of the pion exchange in $2 \pi \mathrm{E}-3 \mathrm{NP}$ at large scattering angles might be suppressed by using the cutoff parameter of $660 \mathrm{MeV}$.

Next, I will examine the BR07-3NP with a large value of $\Lambda$, namely $1000 \mathrm{MeV}$. When the cutoff mass $\Lambda$ larger than $660 \mathrm{MeV}$ is used, calculated ${ }^{3} \mathrm{He}$ binding energy for AV18 $+\mathrm{BR}_{\Lambda}$ are too large. In order to reproduce the ${ }^{3} \mathrm{He}$ binding energy, I introduce a spin-independent 

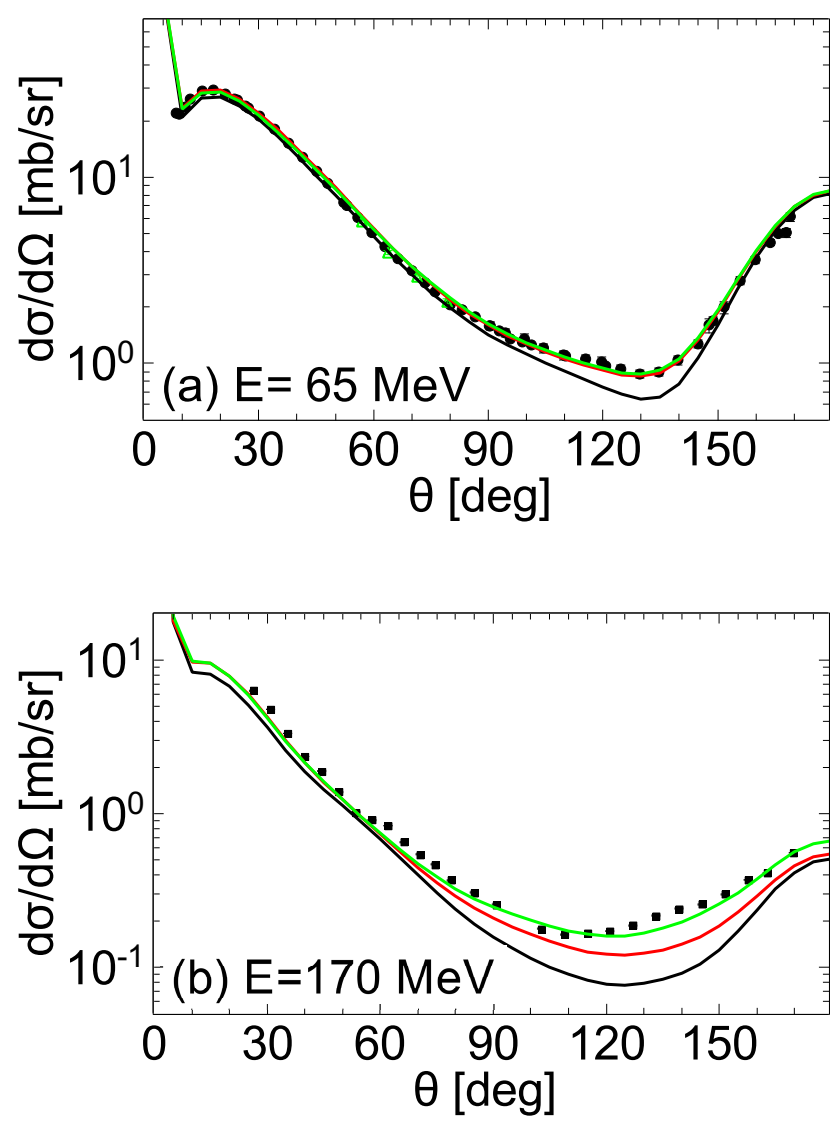

Figure 2: Cross sections for $p$ - $d$ elastic scattering at (a) $E_{p}=65 \mathrm{MeV}$ and (b) $E_{p}=170 \mathrm{MeV}$. Black, red, and green curves denote AV18, AV18+BR 660 , and AV18+BR(C) ${ }_{1000}$ calculations, respectively. Experimental data are taken from Ref. [7] for $E_{p}=65 \mathrm{MeV}$ and Ref. [8] for $E_{p}=170 \mathrm{MeV}$.

repulsive Gaussian 3NP [9], which will be denoted as C-3NP:

$$
W_{\mathrm{C}}=V_{\mathrm{C}} \sum_{\text {cyclic }} e^{-\left(\frac{r_{i k}}{r_{\mathrm{C}}}\right)^{2}-\left(\frac{r_{j k}}{r_{\mathrm{C}}}\right)^{2}}
$$

where $r_{i j}$ is the relative distance between nucleons $i$ and $j, r_{\mathrm{C}}$ the range parameter, and $V_{\mathrm{C}}$ the strength parameter. With fixing $r_{\mathrm{C}}=1.0 \mathrm{fm}$, fitted value of $V_{\mathrm{C}}$ for $\Lambda=1000 \mathrm{MeV}$ is shown as $\mathrm{BR}(\mathrm{C})_{1000}$ in Table 1.

In Fig. 2, calculations of AV18+BR(C) 1000 are shown by green curves, which demonstrates that the introduction of the $\mathrm{BR}(\mathrm{C})_{1000}$ gives almost the same effect on the cross section as $\mathrm{BR}_{660}$ at $E_{p}=65 \mathrm{MeV}$, while increases the cross section at backward angles larger than $\mathrm{BR}_{660}$ to give a reasonable agreement with the data at $E_{p}=170 \mathrm{MeV}$.

\subsection{Inclusive breakup cross section}

Next, I will study the effect of a large cutoff parameter in the $2 \pi \mathrm{E}-3 \mathrm{NP}$ on deuteron breakup reactions, $p+d \rightarrow p+p+n$. Since there are large numbers of configurations for final threenucleon states, here, I just pick up an inclusive breakup reaction, ${ }^{2} \mathrm{H}\left(p, p^{\prime}\right) p n$, which is considered to be a continuation of the elastic scattering. 


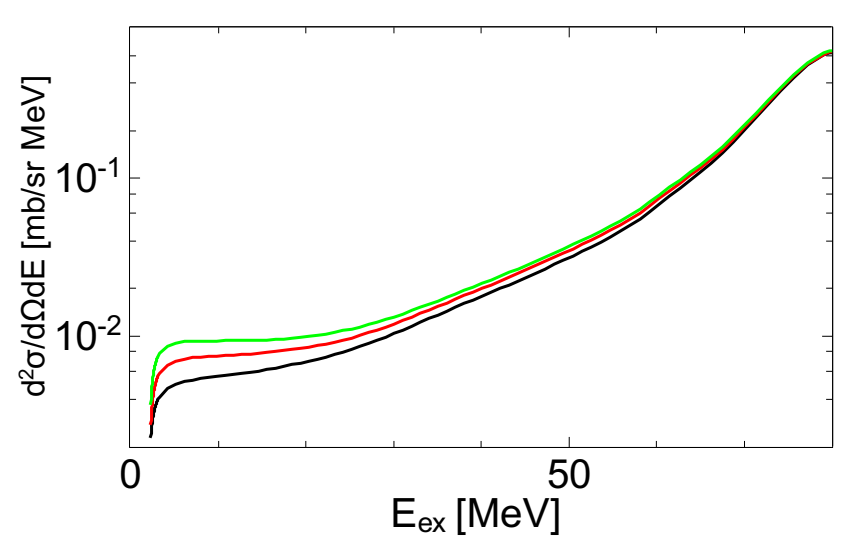

Figure 3: Cross section for inclusive breakup reaction, ${ }^{2} \mathrm{H}\left(p, p^{\prime}\right) p n$, for proton scattering angle $110^{\circ}$ at $E_{p}=170 \mathrm{MeV}$ as a function of excitation energy of the residual $p n$ system. Black, red, and green curves denote AV18, AV18+BR 660 , and AV18+BR(C) ${ }_{1000}$ calculations, respectively.

In Fig. 3, cross sections of the ${ }^{2} \mathrm{H}\left(p, p^{\prime}\right) p n$ reaction for proton scattering angle in laboratory system to be $110^{\circ}$ at $E_{p}=170 \mathrm{MeV}$. This angle corresponds to $140^{\circ}$ at c.m. system in the $p$ - $d$ elastic scattering, where the effect the $\mathrm{BR}(\mathrm{C})_{1000}-3 \mathrm{NP}$ is remarkable as shown in Fig. 2 (b). The cross sections for AV18, AV18+BR 660 , and AV18+BR(C) 1000 are plotted as functions of the excitation energy of the residual $p-n$ system $E_{\text {ex }}$, which is 0 for the elastic scattering. Effects of the large cutoff parameter in the $2 \pi \mathrm{E}-3 \mathrm{NP}$, which are shown by the difference between the red curve and the green curve are well visible.

\subsection{Cutoff in coordinate space}

Since the use of the dipole form factor is not unique way to suppress a short-range part of interactions, I will examine other method: one used in recent the chiral effective field theory ( $\chi$ EFT). In recent calculations of few- and many-nuclear systems [10-12], 2NPs based on $\chi$ EFT are provided in the coordinate space representation with multiplying a damping function (called as regulator) $f_{R}(r)$, where $R$ is a parameter representing the range of cutoff. The cutoff procedure used in constructing the $2 \mathrm{NP}$ is also applied to $3 \mathrm{NP}$ as follows:

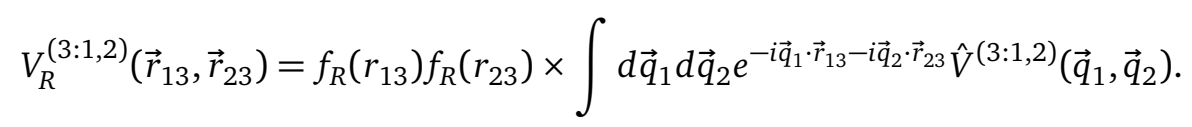

In the present work, two different functional forms of the regulator will be used. One, which will be designated as [I], is from Refs. [10,11]:

$$
f_{R}^{[\mathrm{I}]}(r)=\left[1-\exp \left(-\frac{r^{2}}{R^{2}}\right)\right]^{6},
$$

and the other, which will be designated as [II], is from Ref. [12]:

$$
f_{R}^{[\mathrm{III}]}(r)=1-\frac{1}{(r / R)^{6} e^{(r-R) /(R / 2)}+1} .
$$

First, the range parameters are decided to reproduce the ${ }^{3} \mathrm{He}$ binding energy, and the results with the BR07-3NP are $R=0.87 \mathrm{fm}$ for [I] and $R=1.29 \mathrm{fm}$ for [II]. The regulators with these parameters as functions of $r$ are plotted by black solid curve for $f_{R}^{[\mathrm{I}]}(r)$, and black 


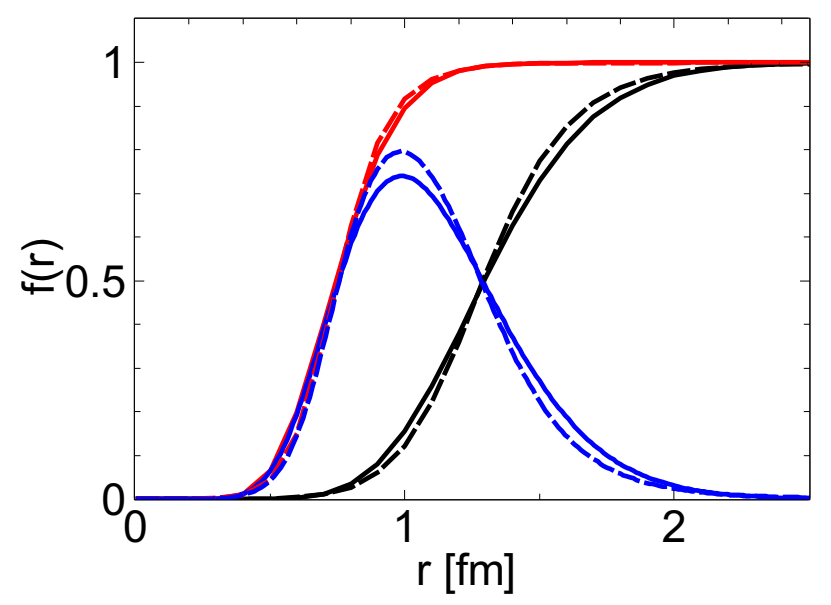

Figure 4: The regulator functions, Eq. (6) and Eq. (7). The meaning of the curves are described in the text.

dashed curve for $f_{R}^{[\mathrm{I}]}(r)$ in Fig. 4. Range of the regulator may be characterized by a value of $r_{0.5}$ that satisfies $f_{R}\left(r_{0.5}\right)=0.5$. For both of $f_{R=0.87}^{[\mathrm{I}]}(r)$ and $f_{R=1.29}^{[\mathrm{II}]}(r), r_{0.5}$ becomes about 1.3 $\mathrm{fm}$, which indicates a long-range character of the regulators.

Next, in order to make the BR07-3NP more attractive, the range parameter $R$ for each of the regulator is changed to be smaller value so that $r_{0.5} \approx 0.75 \mathrm{fm}$, which gives $R=0.5 \mathrm{fm}$ for [I] and $R=0.75 \mathrm{fm}$ for [II]. As in the case of the dipole form factor, I will introduce the repulsive $\mathrm{C}-3 \mathrm{NP}$ to reproduce the ${ }^{3} \mathrm{He}$ binding energy. The strength parameters of the $\mathrm{C}-3 \mathrm{NP}$ are shown as $\mathrm{BR}(\mathrm{C})_{[\mathrm{I}] 0.5}$ and $\mathrm{BR}(\mathrm{C})_{[\mathrm{II}] 0.75}$ in Table 1.

In Fig. 5, calculations of $p$-d scattering at $E_{p}=170 \mathrm{MeV}$ for AV18+BR(C) $[\mathrm{I}] 0.5$, $\mathrm{AV} 18+\mathrm{BR}(\mathrm{C})_{[\mathrm{II}] 0.75}$ as well as $\mathrm{AV} 18+\mathrm{BR}(\mathrm{C})_{1000}$ are plotted. This figure shows a similarity among $\operatorname{AV} 18+B R(C)_{[1] 0.5}, A V 18+B R(C)_{[11] 0.75}$, and $\operatorname{AV} 18+B R(C)_{1000}$ calculations, and these calculations almost equally resolve the difference between the data and calculations. This indicates that the regulator functions $F_{R=0.5}^{[\mathrm{I}]}(r)$ and $F_{R=75}^{[\mathrm{II}]}(r)$ play a similar role as the form factor with $\Lambda=1000 \mathrm{MeV}$ in the $2 \pi \mathrm{E}-3 \mathrm{NP}$.

In Fig. 4, the functions $F_{R=0.5}^{[\mathrm{I}]}(r)$ and $F_{R=0.75}^{[\mathrm{II}]}(r)$ are plotted as red solid curve and red dashed curve, respectively. In the figure, the differences between $F_{R=0.5}^{[\mathrm{I}]}(r)$ and $F_{R=0.87}^{[\mathrm{I}]}(r)$, and $F_{R=0.75}^{[\mathrm{II}]}(r)$ and $F_{R=1.29}^{[\mathrm{III}]}(r)$ are plotted as blue solid curve and blue dashed curve, respectively, which demonstrates that the increase of the $p$ - $d$ cross sections at minimum and backward angle regions is caused by effects of the pion-exchange around $r=1 \mathrm{fm}$.

\section{Conclusion}

The use of a larger value of cutoff parameter in the form factor, or a short range cutoff of the regulator in the $2 \pi \mathrm{E}-3 \mathrm{NP}$ enhances $p$ - $d$ elastic cross sections at backward angles for intermediate energies, which tends to reduce the discrepancies between data and calculations. Similar effects are observed in inclusive breakup reactions. This demonstrates the importance of pion exchange in the $2 \pi \mathrm{E}-3 \mathrm{NP}$ around $r=1 \mathrm{fm}$.

In this work, the repulsive $3 \mathrm{NP}$ to compensate the large attractive effect of the $2 \pi \mathrm{E}-3 \mathrm{NP}$ is introduced phenomenologically. Physical origin of it as well as spin-dependence of 3NPs to reproduce polarization observables in $p$ - $d$ scattering are left as future problems. 


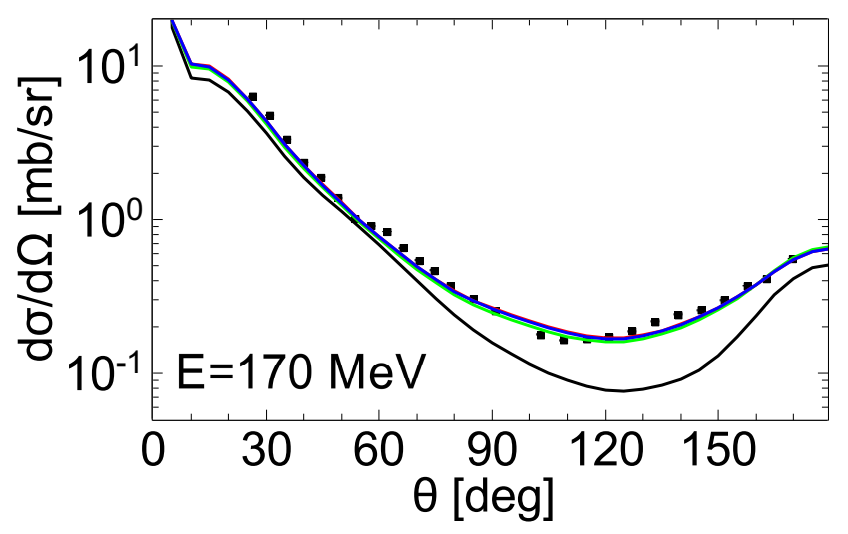

Figure 5: Cross sections for $p$ - $d$ elastic scattering at $E_{p}=170 \mathrm{MeV}$. Black, green, blue, and cyan curves denote AV18, AV18+BR(C) $1000, A V 18+\operatorname{AV} 18+B R(C)_{[1] 0.5}$, and AV18+BR(C) $[\mathrm{II}] 0.75$ calculations, respectively. The latter three curves are almost overlapped mutually. Experimental data are taken from Ref. [8].

\section{References}

[1] S. Ishikawa, Two-pion exchange three-nucleon force effects in elastic nucleon-deuteron scattering cross sections, Few-Body Syst. 60, 39 (2019), doi:10.1007/s00601-019-1506-z.

[2] L. D. Faddeev, Scattering theory for a three-particle system, Zh. Eksp. Teor. Fiz. 39, 1459 (1961) http://www.jetp.ac.ru/cgi-bin/e/index/e/12/5/p1014?a=list.

[3] S. Ishikawa, Coordinate space proton-deuteron scattering calculations including Coulomb force effects, Phys. Rev. C 80, 054002 (2009), doi:10.1103/PhysRevC.80.054002.

[4] S. Ishikawa and M. R. Robilotta, Two-pion exchange three-nucleon potential: $O\left(q^{4}\right)$ chiral expansion, Phys. Rev. C 76, 014006 (2007), doi:10.1103/PhysRevC.76.014006.

[5] R. B. Wiringa, V. G. J. Stoks and R. Schiavilla, Accurate nucleon-nucleon potential with charge-independence breaking, Phys. Rev. C 51, 38 (1995), doi:10.1103/PhysRevC.51.38.

[6] R. Machleidt, High-precision, charge-dependent Bonn nucleon-nucleon potential, Phys. Rev. C 63, 024001 (2001), doi:10.1103/PhysRevC.63.024001.

[7] H. Shimizu, K. Imai, N. Tamura, K. Nisimura, K. Hatanaka, T. Saito, Y. Koike and Y. Taniguchi, Analyzing powers and cross sections in elastic p-d scattering at $65 \mathrm{MeV}$, Nucl. Phys. A 382, 242 (1982), doi:10.1016/0375-9474(82)90134-8.

[8] K. Ermisch et al., Systematic investigation of the elastic proton-deuteron differential cross section at intermediate energies, Phys. Rev. C 68, 051001 (2003), doi:10.1103/PhysRevC.68.051001.

[9] S. Ishikawa, M. Tanifuji and Y. Iseri, Central and tensor components of three-nucleon forces in low-energy proton-deuteron scattering, Phys. Rev. C 67, 061001 (2003), doi:10.1103/PhysRevC.67.061001.

[10] S. Binder et al., Few-nucleon and many-nucleon systems with semilocal coordinatespace regularized chiral nucleon-nucleon forces, Phys. Rev. C 98, 014002 (2018), doi:10.1103/PhysRevC.98.014002. 
[11] E. Epelbaum et al., Few- and many-nucleon systems with semilocal coordinate-space regularized chiral two- and three-body forces, Phys. Rev. C 99, 024313 (2019), doi:10.1103/PhysRevC.99.024313.

[12] M. Piarulli et al., Light-nuclei spectra from chiral dynamics, Phys. Rev. Lett. 120, 052503 (2018), doi:10.1103/PhysRevLett.120.052503. 